\title{
Numerical modeling of air treatment processes in contact apparatuses
}

\author{
Vera Ulyasheva ${ }^{1, *}$ and Nikita Kryshkin $^{1}$ \\ ${ }^{1}$ Saint Petersburg State University of Architecture and Civil Engineering, 190005 St. Petersburg, \\ Russia
}

\begin{abstract}
Formation of standardized parameters of air in rooms largely depends on climatic features of the area. It is known that at low ambient temperatures (below $-15^{\circ} \mathrm{C}$ ) and with standard heating and ventilation systems in rooms, relative air humidity in rooms is about $5 \%$ with the minimum of $15 \%$. There is an issue related to determination of the efficiency of heat-and-moisture treatment of air in chambers with irrigated porous packing, which depends on such factors as the geometric shape of channels, hydrodynamic conditions for interaction of the wetted surface of the packing, water film flowing down the surface of the irrigated packing, and air flow moving towards the water film. The present paper presents results of numerical modeling for heat and mass transfer processes using the STAR-CCM+ software package. The k- $\varepsilon$ model was used as the turbulence model. Research data can be used to assess quantitative characteristics in contact apparatuses of various configurations.
\end{abstract}

\section{Introduction}

A number of works have been devoted to improving the methods for calculating heat and mass transfer processes in contact apparatuses of air conditioning systems, including domestic and foreign scientists - E.V. Stefanov, V.S. Maysotsenko, A.G. Sotnikov, O.Ya. Kokorin, S.M. Anisimov, M.G. Tarabanov, A.G. Averkin, A.N. Gvozdkov, D. Pandelidis and others [1-8]. In these studies, of particular importance is the use of adiabatic processes, which can be used both for humidification of air in the cold period and evaporative cooling of air in the warm season. Such processes are highly relevant not only for Russia. Nowadays, spray decks and honeycomb humidifiers are most widely used among contact apparatuses. There is a wide variety of honeycomb humidifiers both in terms of the materials used and the shape of the nozzles, as well as the methods of supplying water. The complex nature of the interaction of air flows, water mist or a flowing water film between each other and with a solid wetted surface leads to the fact that in most cases, the results of experimental studies are used for engineering calculations. In addition, in the process of distributing water over a heat and mass transfer surface, some of the water is used to wet the surface, and as it drains, the film thickness decreases due to the simultaneous evaporation of water. In this case, water film sections with both laminar and turbulent flow

\footnotetext{
*Corresponding author: author@email.org
} 
regimes form on the wall [9]. Given the changes in almost all parameters of water and air, such as fluid flow rate, irrigation density, Reynolds number, relative air humidity, it becomes relevant to use modern numerical methods to solve a complex system of differential equations describing the processes of heat-moisture treatment of air when it comes in contact with flowing liquid water film.

\section{Materials and Methods}

Modern methods of mathematical modeling using applied programs are used in various fields of science and technology, including in studies of heat transfer processes in confined spaces [10-13]. Some results of numerical modeling of heat and mass transfer processes upon contact of air with a water film are presented in $[9,14,15]$. As mentioned above, heat and mass transfer processes in this case depend on a significant number of geometric and physical factors, among which, first of all, it is necessary to note the shape of the channels in the porous structure of the irrigated nozzle. The study adopted the well-known nozzle of Munters design, having the following parameters: 60 corrugated sheets (wavelength - 17 $\mathrm{mm}$ ) placed at angles of $45^{\circ}$ up and $15^{\circ}$ down to the horizon (Fig. 1).

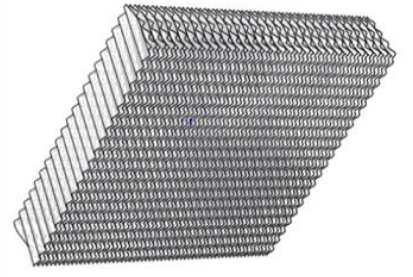

General form

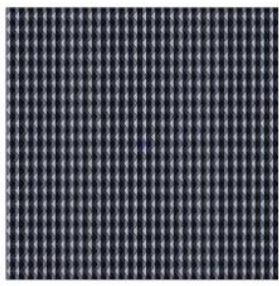

Characteristic section

Fig. 1. Heat and mass transfer nozzle.

To select honeycomb apparatuses with such a nozzle, nomograms [9] with limited information are used, which do not allow obtaining detailed characteristics from the point of view of heat and mass transfer and hydroaerodynamics. To ensure uniform aerodynamic characteristics of the treated air at the inlet before and after the irrigated nozzle, sections of the ducts are provided. Using the SolidWorks program, a 3D model of the object under study was created (Fig. 2,3).

When sampling the computational domain, a rectangular non-uniform grid was used, which thickens near solid boundaries according to the law of hyperbolic tangent, the socalled Vinokur algorithm: the base size is $20 \mathrm{~mm}$, in the duct sections before and after the nozzle $-10 \mathrm{~mm}$, in the immediate vicinity of the nozzle plates $-4 \mathrm{~mm}$. The total number of cells in the grid designed in SolidWorks is about 3 million.

For the numerical experiment, the StarCCM+ software package was used. After further transformations using the built-in automatic generators of prismatic layers, surface grid, etc., the total number of cells in the designed grid amounted to more than 5 million. 


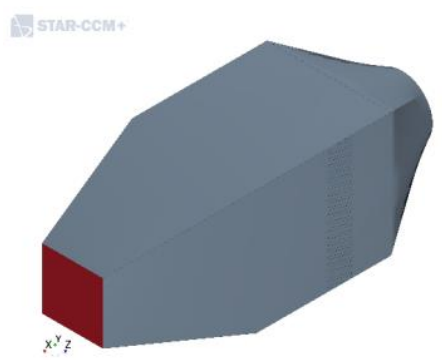

General form

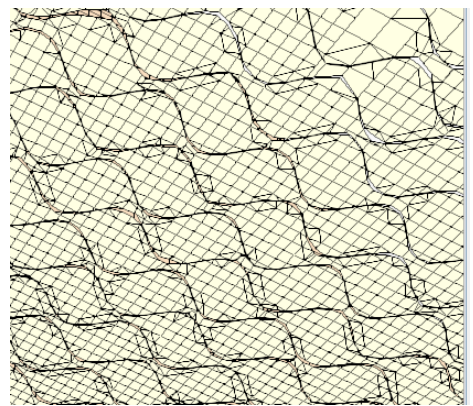

Grid element

Fig.2. To build a geometric model of the installation.

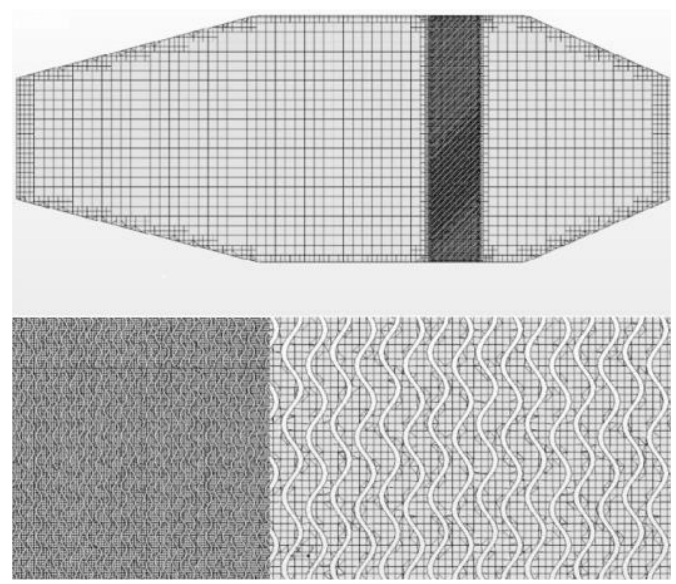

Fig.3. Fragments of the calculated grid.

The numerical simulation of any heat and mass transfer processes using similar hydrodynamic complexes is based on the solution of the Reynolds equations designed to describe turbulent flows, and also the convective-diffusion transfer equations for an averaged scalar substance $\bar{a}$, as which, for example, temperature $\mathrm{T}$ and moisture content $\mathrm{d}$ can be taken:

$$
\begin{aligned}
& \frac{\partial \bar{\rho}}{\partial t}+\frac{\partial}{\partial x_{j}}\left(\bar{\rho} \bar{u}_{i}+\bar{\rho}^{\prime} \bar{u}_{i}^{\prime}\right)=S_{m} \\
& \rho\left(\frac{\partial \bar{u}_{i}}{\partial t}+\bar{u}_{j} \frac{\partial \bar{u}_{i}}{\partial x_{j}}\right)=-\frac{\partial \bar{P}}{\partial x_{j}}+\mu\left(\frac{\partial \bar{u}_{i}}{\partial x_{j}}+\frac{\partial \bar{u}_{i}}{\partial x_{i}}\right)+\frac{\partial \tau_{i j}^{\prime}}{\partial x_{j}}+S_{i} \\
& \frac{\partial(\bar{\rho} \bar{a})}{\partial t}+\frac{\partial\left(\overline{\rho a} \bar{u}_{i}\right)}{\partial x_{j}}=-\frac{\partial\left(\overline{\left.\rho u_{j}^{\prime} a^{\prime}\right)}\right.}{\partial x_{j}}+\bar{J}_{a}
\end{aligned}
$$

where $t-{ }_{\mathrm{t}}$ ime; $\rho$ - density; $\mu-$ dynamic viscosity coefficient; $\bar{u}_{j}-$ components of the averaged velocity vector along the coordinate axes; $\tau_{i j}-$ turbulent stresses (additional Reynolds stresses); $u_{i}^{\prime}, u_{j}^{\prime}, T^{\prime}, C^{\prime}-$ local ripple of velocity, temperature and impurity 
flow; $\bar{a}$ - averaged specific gravity of a scalar quantity; $S_{m}, S_{i}-$ intensity of mass and impulse sources; $\bar{J}_{a}-$ intensity of impurity sources.

This work provides for the modeling of turbulent air flow through an irrigated nozzle with the simultaneous adiabatic process of heat and mass transfer and laminar motion of a liquid film on the wetted surface of the nozzle under the action of gravitational forces. The calculation adopted the following: stationary heat and mass transfer process, ideal gas, surface-to-surface radiation, multicomponent gas. When modeling, the influence of gravity was taken into account. The $\mathrm{k}-\varepsilon$ turbulence model is used. To account for the features of the process of evaporation of a liquid film, the Eulerian multiphase model is used. To find the pressure of saturated water vapor, the Antoine equation is used.

The boundary conditions are the speed, temperature, and relative humidity of the air at the entrance to the object under study; stationary temperature of the surface of the walls of the installation.

The transition from partial differential equations to discrete ones is carried out by the finite volume method. When conducting a numerical experiment, the convergence was estimated, which means that the errors in the numerical solution are reduced to zero, provided that the sizes of the control volumes of the computational domain tend to zero. The calculation is considered complete if the behavior of the studied parameters becomes stationary (Fig. 4).

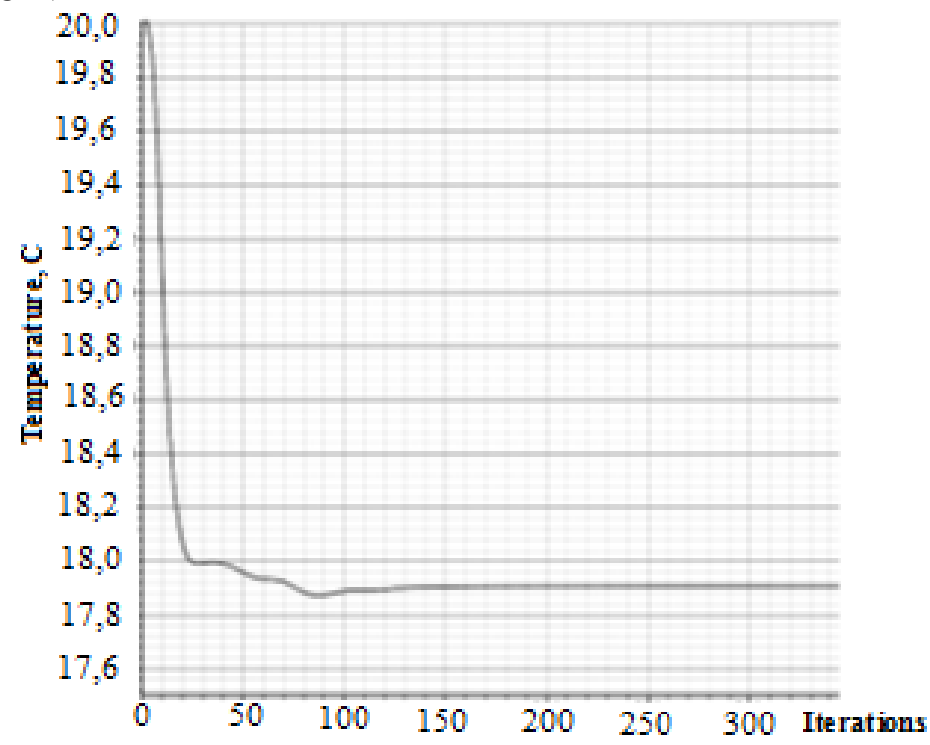

Fig.4. The convergence of air temperature.

\section{Results and Discussion}

The results of a numerical experiment are presented in Fig. 5 - 9 in the form of fields of velocity, temperature and moisture content of air in general form and characteristic secant planes. 


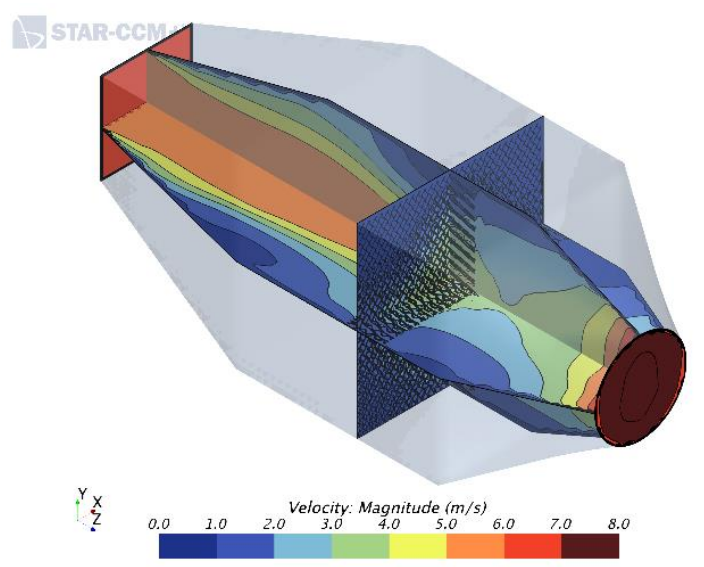

Fig.5. The distribution of air velocity in the elements of the object.

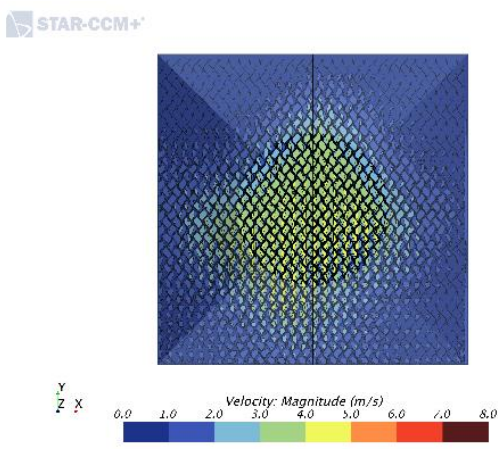

Fig.6. The distribution of air velocity in the cross section of the heat and mass transfer nozzle.

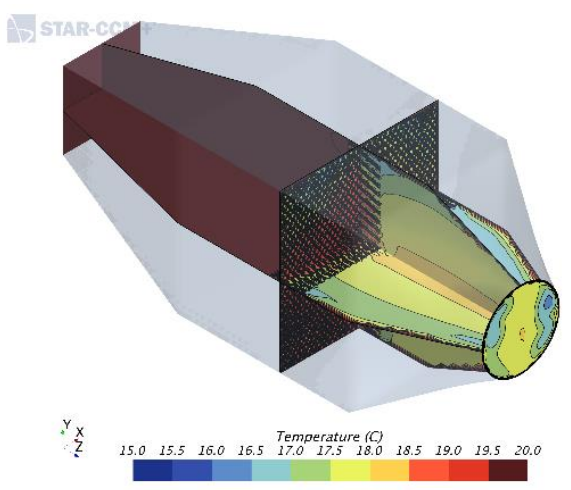

Fig.7. The distribution of air temperature in the elements of the object. 


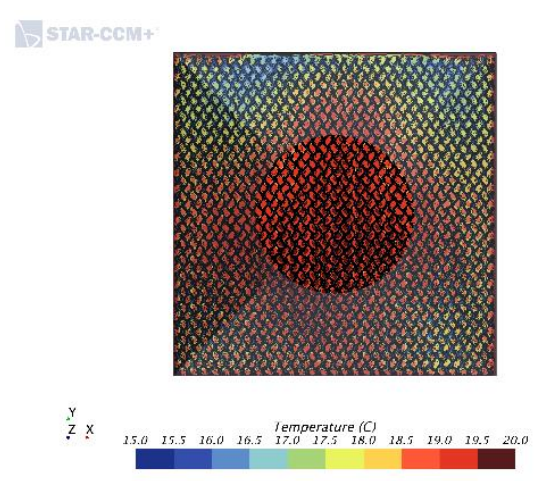

Fig.8. The distribution of air temperature in the cross section of the heat and mass transfer nozzle.
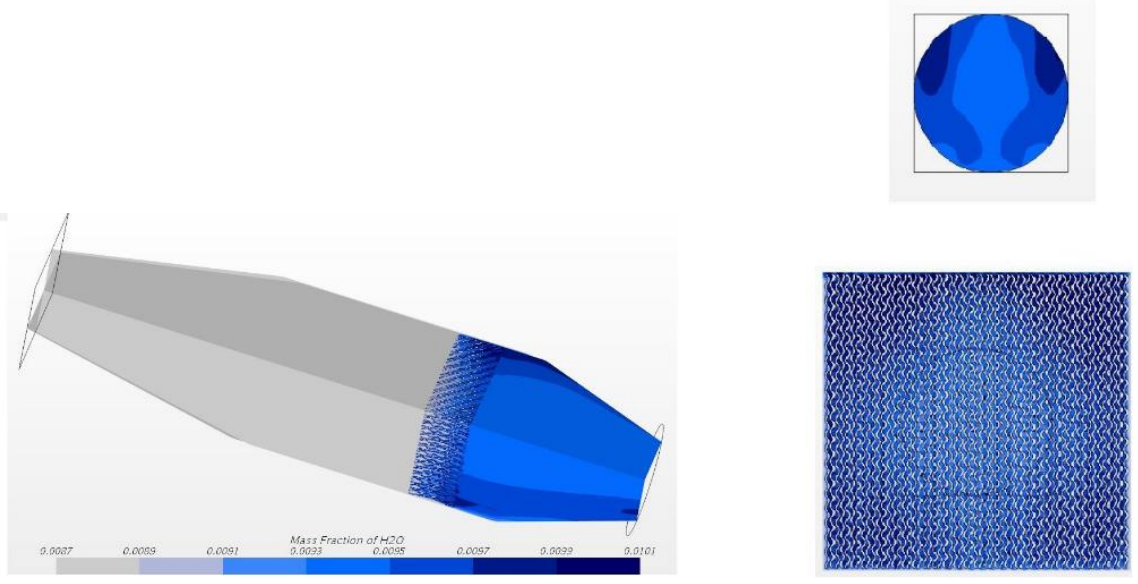

Fig.9. The distribution of moisture content of air $(\mathrm{kg} / \mathrm{kg})$ in the elements of the object.

Figure 10 shows the distribution of the thickness of the liquid film on a fragment of the irrigated surface.

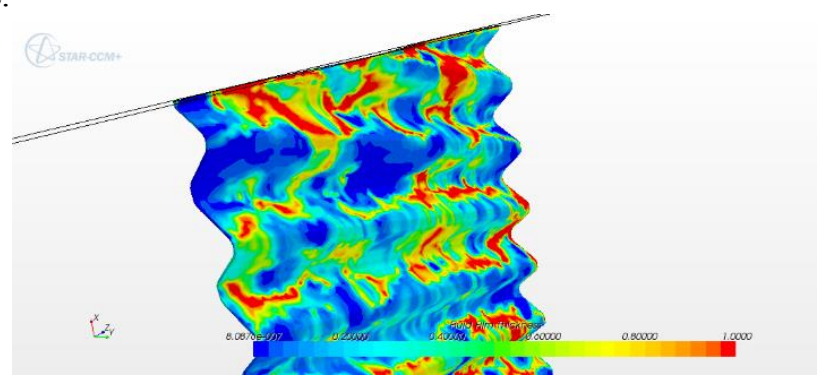

Fig.10. The distribution of the thickness of the liquid film on the fragment of the irrigated surface.

Based on the results of a numerical experiment, the coefficients of adiabatic efficiency of the heat and mass transfer process in the irrigated nozzle are determined. So, when the air velocity in the clear air of the nozzle is $1.4 \mathrm{~m} / \mathrm{s}$; the initial air temperature of $20^{\circ} \mathrm{C}$ and the final temperature of $17.2^{\circ} \mathrm{C}$, respectively; water temperature of $15.1{ }^{\circ} \mathrm{C}$, the average value of the coefficient of adiabatic efficiency in terms of temperature and moisture content $\mathrm{EA}=58 \%$ (air flow $\mathrm{G}=800 \mathrm{~m}^{3} / \mathrm{h}$ ). The calculation results correspond to the data of a laboratory experiment performed by the authors (Fig. 11). 


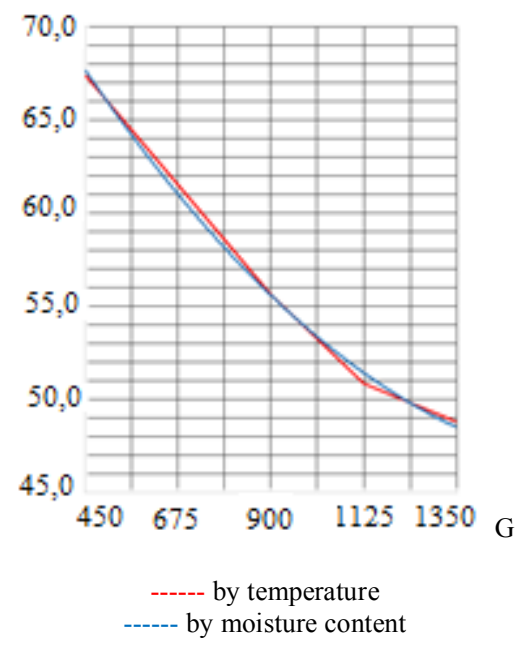

Fig.11. The dependence of the adiabatic efficiency coefficient of air flow.

\section{Conclusions}

Based on the developed numerical model on the basis of the k- $\varepsilon$ turbulence model, detailed characteristics of heat and mass transfer processes during the evaporation of a liquid film in a honeycomb humidifier are obtained. Due to the design features of the nozzle and the flow of the liquid film, a certain uneven distribution of parameters is observed. Nevertheless, there is a correspondence between the average values of the air parameters, as well as the coefficient of adiabatic efficiency of the treatment process in the nozzle under study, with the results of laboratory experiments.

\section{References}

1. Ye.V. Stefanov, (AVHK North-West, St. Petersburg, 2005)

2. V.S. Maysotsenko, J.Ind.heat eng. 9, 2, 84-87 (1987)

3. A. G. Sotnikov, Proc.app.syst. 2, 2 (2007)

4. O.Ya. Kokorin, AVHK 2, 46-48 (2011)

5. M. G. Tarabanov AVHK - North-West 3, 41 (2009)

6. S.M. Anisimov, V.I Polushkin, D. Pandelidis, 4 (2012)

7. A.G. Averkin, (Publishing House Lan. Russia, 2016)

8. A. N. Gvozdkov, J Constr.arch., 2, 133-142 (2014)

9. V.F. Vasilyev, V.M. Ulyasheva, M.A. Kanev, J Pl.H.Ven. 12, 180, 66-69 (2016)

10. H.-S. Dou, G. Jiang, L. Chengwang, DOI: 10.1155/2013/198695 (2013)

11. M. Muia, K. Awuor, J Nat.Sc.Res. 8, 6 (2018)

12. S. de Ornelas, S. de Sousa, C. Dong and et al., http://www.math.sjsu.edu/camcos/samples/2005-2006.spring.intel.pdf (2006)

13. A.M. Grimitlin. T.A., Datsyuk, (AVHK North-West, St. Petersburg, 2013)

14. D. Cherrared, J Th. Eng., 3, 2, 1110-1120 (2017)

15. G.M. Pozin, V.M Ulyasheva, N.M. Kryshkin, J. Pl.H.V. 2, 62-65. (2019) 